\title{
An Analysis of the methods of solving the finite sequence of convolution sum and circular convolution Huajun Chen ${ }^{1, a}$, Lina Yuan ${ }^{1, b}$

\author{
${ }^{1}$ School of Information Engineering, Tongren University, Guizhou ,554300, China \\ a390832683@qq.com, b893422817@qq.com
}

Keywords: convolution sum; circular convolution; vertical multiplication

\begin{abstract}
The calculation of finite length sequences of convolution sum and circular convolution plays a significant role in the courses of "signal and system" and "digital signal processing".This paper mainly introduces three kinds of the methods of solving the finite sequence of convolution and and circular convolution. For both,vertical multiplication is easy to learn, and is advantageous to the student's understanding.
\end{abstract}

\section{Introduction}

In the courses of "signal and system" and "digital signal processing",general books only introduce to use formula method and graphical method of solving finite length sequences of convolution sum and circular convolution. Though the two methods of physical concepts are clear, their calculation is rather complicated, time-consuming and error-prone. This paper avoids the two methods, and mainly introduces tabulation method、vertical multiplication and Matlab to solve the finite length sequence convolution sum and the circular convolution respectively.

\section{The computing method of finite length sequence convolution sum}

\subsection{To use tabulation method of solving the finite length sequence convolution sum}

Put respectively the values of $\mathrm{h}[\mathrm{k}]$ and $\mathrm{f}[\mathrm{k}]$ to be in line and a list orderly. The intersection of a row and column writes down the corresponding multiplication of $f[k]$ and $h[k]$.As shown in Fig. 1 , note that the numerical values of the diagonal slash are the values of $f[n]$ multiplying $h[k-n]$, the sum of the numerical values of the diagonal slash is the corresponding values of $y[\mathrm{k}]$.

For example,compute the convolution sum of $h[k]=\{1,2,0,3,2\}_{0}$ and $f[k]=\{1,2,2,3\}_{0}$. According to the tabulation method, list as shown in Fig. 2, thus work out the circulation sum of the sequences $\mathrm{f}[\mathrm{k}]$ and $\mathrm{h}[\mathrm{k}]$, that is $\mathrm{y}[\mathrm{k}]$ equating $\{1,4,6,10,14,10,13,6\}_{0}$.

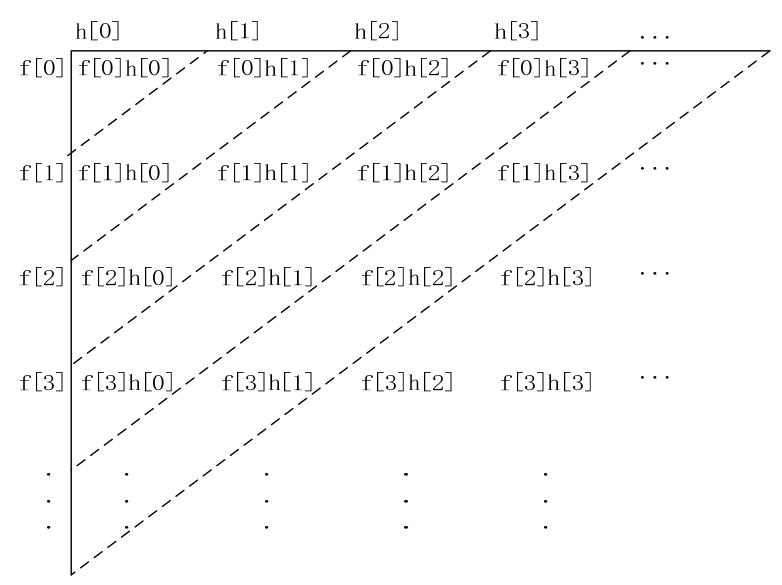

Fig.1 The tabulation method of solving

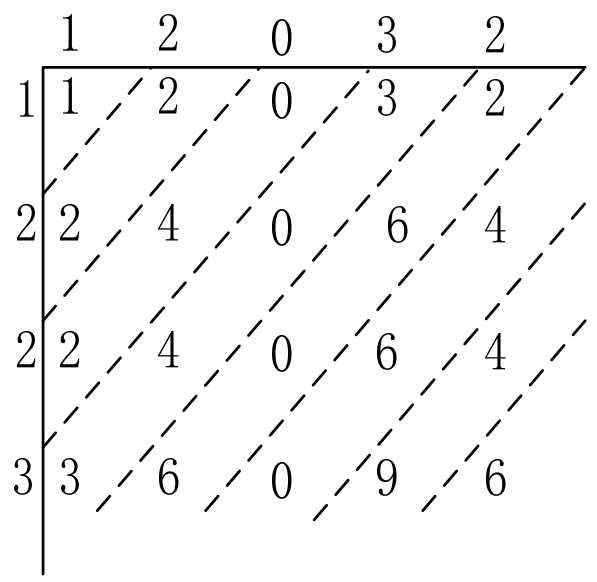

Fig. 2 Tabulation method the finite length sequence convolution sum 


\subsection{To use vertical multiplication of solving the finite length sequence convolution sum}

Solving the finite length sequence convolution sum according to the order "from right to left".Firstly, list vertical form, just like multiplication-tail alignment and use the numerical values of the next line to multiply the last row but do not carry. Secondly, add the same column multiplier and also do not carry.Lastly,readings can be read from left to right.For example,compute the convolution sum of $\mathrm{h}[\mathrm{k}]=\{1,2,0,3,2\}_{0}$ and $\mathrm{f}[\mathrm{k}]=\{1,2,2,3\}_{0}$. According to the method, list as shown in Fig. 3, thus work out the circulation sum of the sequences $\mathrm{f}[\mathrm{k}]$ and $\mathrm{h}[\mathrm{k}]$,that is $\mathrm{y}[\mathrm{k}]$ equating $\{1,4,6,10,14,10,13,6\}_{0}$. The computing result is the same as the tabulation method.

\begin{tabular}{llllllll} 
& & & 1 & 2 & 0 & 3 & 2 \\
& & & 1 & 2 & 2 & 3 \\
\cline { 3 - 8 } & & & 3 & 6 & 0 & 9 & 6 \\
& 2 & 4 & 0 & 6 & 4 & \\
& 2 & 4 & 0 & 6 & 4 & & \\
1 & 2 & 0 & 3 & 2 & & & \\
\hline 1 & 4 & 6 & 10 & 14 & 10 & 13 & 6
\end{tabular}

Fig. 3 vertical multiplication-from right to left

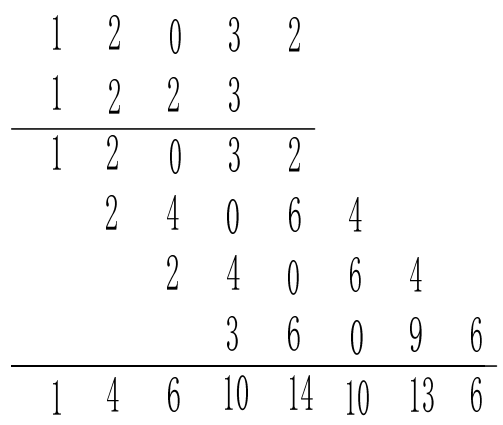

Fig.4 vertical multiplication-from left to right

Solving the finite length sequence convolution sum according to the order "from left to right".Firstly, list vertical form-left justifying(first alignment) and use the numerical values of the next line to multiply the last row but do not carry. Multiply from the left, and each line needs to shift to the right one.Secondly, add the same column multiplier and also do not carry.Lastly,readings can be read from left to right. For example,compute the convolution sum of $\mathrm{h}[\mathrm{k}]=\{1,2,0,3,2\}_{0}$ and $\mathrm{f}[\mathrm{k}]=\{1,2,2,3\}_{0}$. According to the method, list as shown in Fig. 4, thus work out the circulation sum of the sequences $\mathrm{f}[\mathrm{k}]$ and $\mathrm{h}[\mathrm{k}]$,that is $\mathrm{y}[\mathrm{k}]$ equating $\{1,4,6,10,14,10,13,6\}_{0}$. The computing result is the same as the above two methods.

To use Matlab of solving the finite length sequence convolution sum. Take computing the convolution sum of $\mathrm{h}[\mathrm{k}]=\{1,2,0,3,2\}_{0}$ and $\mathrm{f}[\mathrm{k}]=\{1,2,2,3\}_{0}$ as an example. Firstly,compile the two child functions-cirshift.m and circonvt.m in the $\mathrm{M}$ - file, and their codes list respectively as follows:

function $\mathrm{y}=\operatorname{cirshift}(\mathrm{h}, \mathrm{m}, \mathrm{N})$

if length $(h)>N$

error('N must be equal or greater than the

length of $h ')$

end

$\mathrm{h}=[\mathrm{h}$ zeros $(1, \mathrm{~N}-$ length $(\mathrm{h}))]$;

$\mathrm{n}=[0: 1: \mathrm{N}-1]$;

$\mathrm{n}=\bmod (\mathrm{n}-\mathrm{m}, \mathrm{N})$;

$\mathrm{y}=\mathrm{h}(\mathrm{n}+1)$;

function $\mathrm{y}=\operatorname{circonvt}(\mathrm{f}, \mathrm{h}, \mathrm{N})$

if length(f) $>\mathrm{N}$

error('Nmust be equal or greater than the length of $f^{\prime}$ )

end

if length(h) $>\mathrm{N}$

end

error('N must be equal or greater than the length of h')

$\mathrm{f}=[\mathrm{f} \operatorname{zeros}(1, \mathrm{~N}$-length(f)) $]$;

$\mathrm{h}=[\mathrm{h}$ zeros $(1, \mathrm{~N}$-length $(\mathrm{h}))]$;

$\mathrm{m}=[0: 1: \mathrm{N}-1]$;

$\mathrm{h}=\mathrm{h}(\bmod (-\mathrm{m}, \mathrm{N})+1)$;

$\mathrm{H}=\operatorname{zeros}(\mathrm{N}, \mathrm{N})$;

for $\mathrm{n}=1: 1: \mathrm{N}$ 
end

$H(n,:)=\operatorname{cirshift}(h, n-1, N)$;

$\mathrm{y}=\mathrm{f}^{*} \mathrm{H}^{\prime}$;

Secondly,input the following codes in the command window of Matlab:

$\mathrm{f}=\left[\begin{array}{lllll}1 & 2 & 0 & 3 & 2\end{array}\right]$;

$\mathrm{h}=\left[\begin{array}{llll}1 & 2 & 2 & 3\end{array}\right]$;

$\mathrm{y}=\operatorname{circonvt}(\mathrm{f}, \mathrm{h}, 8)$

Lastly,get the result-y[k] $=\{1,4,6,10,14,10,13,6\}_{0}$.

\section{The computing method of circular convolution}

\subsection{To use tabulation method of solving circular convolution}

Take computing five points circular convolution of $\mathrm{h}[\mathrm{k}]=\{1,2,0,3,2\} 0$ and $\mathrm{f}[\mathrm{k}]=\{1,2,2,3\} 0$ as an example.List as shown in Fig. 5, thus work out five points circular convolution of the sequences f[k] and $\mathrm{h}[\mathrm{k}]$,that is $\mathrm{y}[\mathrm{k}]$ equating $\{11,17,12,10,14\}_{0}$.

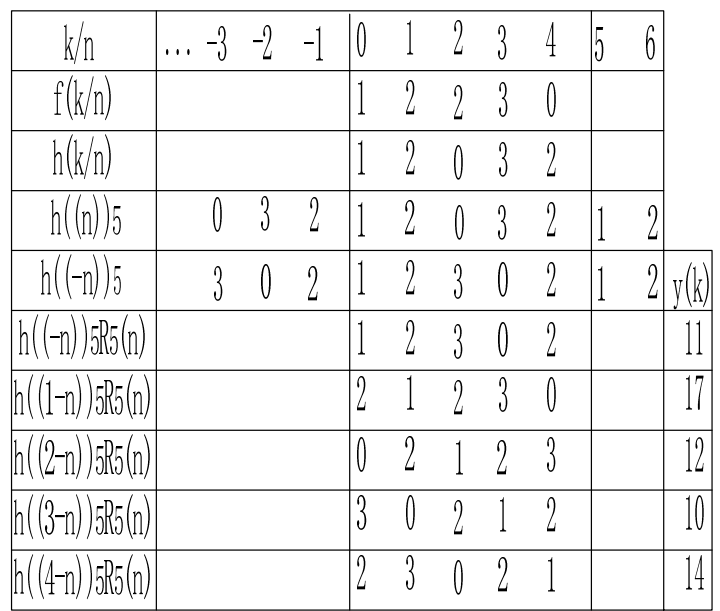

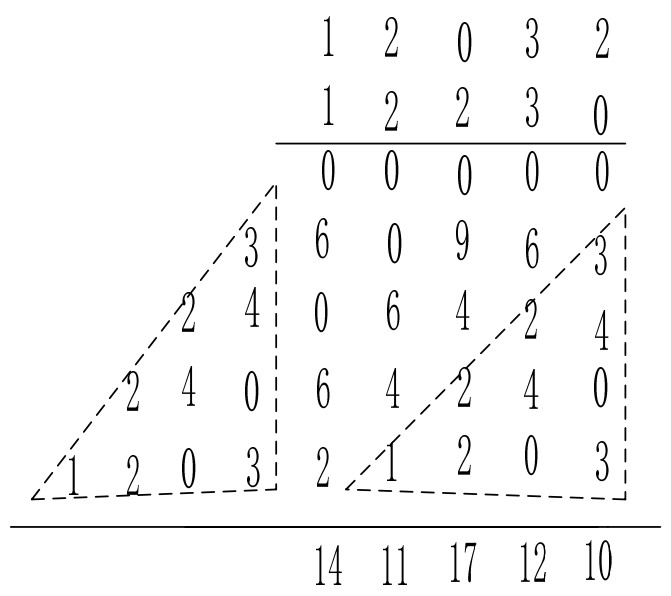

Fig. 5 tabulation method

Fig. 6 vertical multiplication-from right to left

\subsection{To use vertical multiplication of solving circular convolution}

Solving circular convolution according to the order "from right to left" Firstly, list vertical form, just like multiplication-tail alignment and use the numerical values of the next line to multiply the last row but do not carry. Secondly, retain to calculate $\mathrm{N}$ points, draw a triangle respectively in the right and left, and shift the triangle of left to the right.Thirdly, add the same column multiplier and also do not carry.Noting that readings can be read from the left side of the second to the right side, and the left side of the first must be read lastly.For example,compute five points circular convolution of $\mathrm{h}[\mathrm{k}]=\{1,2,0,3,2\}_{0}$ and $\mathrm{f}[\mathrm{k}]=\{1,2,2,3\}_{0}$. According to the method, list as shown in Fig. 6 , thus work out circular convolution of the sequences $f[k]$ and $h[k]$,that is $y[k]$ equating $\{11,17,12,10,14\}_{0}$. The computing result is the same as the tabulation method.

Solving circular convolution according to the order "from left to right". Firstly, list vertical form-left justifying(first alignment) and use the numerical values of the next line to multiply the last row but do not carry. Multiply from the left, and each line needs to shift to the right one.Secondly, retain to calculate $\mathrm{N}$ points, draw a triangle respectively in the right and left, and shift the triangle of right to the left.Thirdly, add the same column multiplier and also do not carry. Noting that readings can be read from left to right.For example,compute five points circular convolution of $\mathrm{h}[\mathrm{k}]=\{1,2,0,3,2\}_{0}$ and $\mathrm{f}[\mathrm{k}]=\{1,2,2,3\}_{0}$. According to the method, list as shown in Fig. 7 , thus work out circular convolution of the sequences $f[k]$ and $h[k]$, that is $y[k]$ equating $\{11,17,12,10,14\}_{0}$. The computing result is the same as the above two methods. 


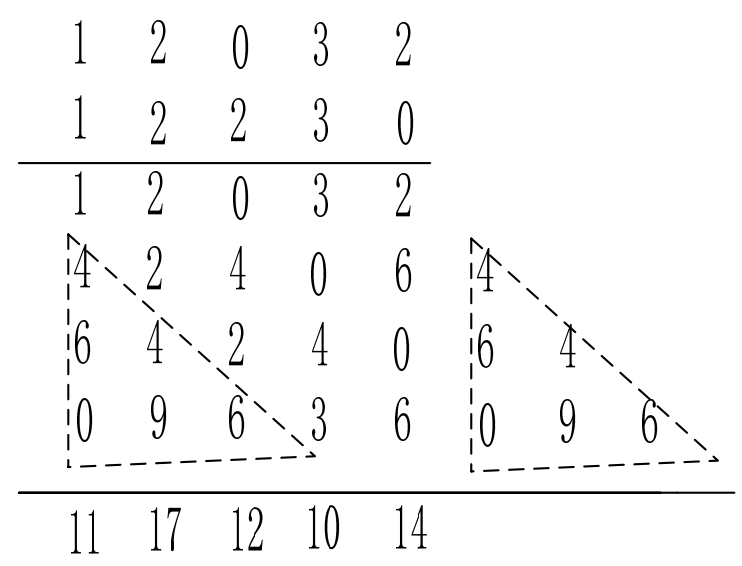

Fig. 7 vertical multiplication-from left to right

\subsection{To use Matlab of solving circular convolution}

Take computing five points circular convolution of $\mathrm{h}[\mathrm{k}]=\{1,2,0,3,2\}_{0}$ and $\mathrm{f}[\mathrm{k}]=\{1,2,2,3\}_{0}$ as an example.Firstly,compile the two child functions-cirshift.m and circonvt.m in the $\mathrm{M}$ - file, and their codes are the same as computing the finite length sequence convolution sum.Secondly, input the following codes in the command window of Matlab:

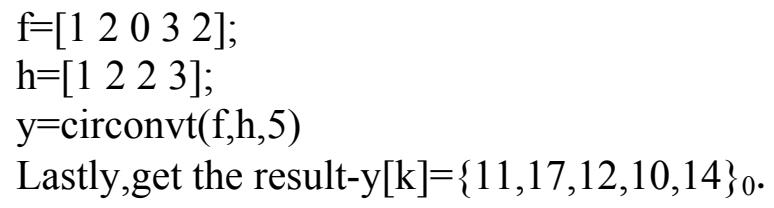

\section{Summary}

From the above discussion, whether to solve the finite sequence of convolution sum or circular convolution, vertical multiplication is relatively simple.As long as the understanding of the finite length sequence of convolution sum and circular convolution, it doesn't discuss all kinds of calculation methods in classroom teaching too much.

\section{Acknowledgment}

This work is supported by the Collaborative Fund Project of Science and Technology Agency in Guizhou Province marked by the word LH on 7487[2014] and the reform project of teaching contents and curriculum system in colleges and universities of Guizhou Province on 2014SJJGXM003.

\section{References}

[1]. Sanjit K.Mitra,Digital Signal Processing: A Computer-Based Approach[M](Third Edition) . BEIJING: Publish House of Electronics Industry,2006,p:157-159。 